\title{
Numeric simulation of aircraft engine parts additive manufacturing process
}

\author{
Peter Maksimov ${ }^{l}$, Oleg Smetannıkov ${ }^{l}$, Aleksandra Dubrovskaya, ${ }^{2}$, , Konstantin \\ Dongauzer ${ }^{2}$, and Leonid Bushuev ${ }^{2}$ \\ ${ }^{1}$ Perm national research polytechnic university, Computation Mathematics and Mechanics \\ Department 29, Komsomolsky prospect, Perm, 614990, Russian Federation \\ ${ }^{2}$ JSC UEC-Aviadvigatel, Simulation department, 93, Komsomolsky ave., Perm, 614990, Russian \\ Federation
}

\begin{abstract}
This paper presents the results of software (ANSYS software) improvements specific for modeling the physical process of SLM (Selective Laser Melting). Improvement goal was to create a set of mathematical models and user environment (a set of APDL programs) based on the ANSYS finite element analysis system solver, allowing to perform the technological procedure of physical SLM process numerical modeling to the required degree of precision with an estimate of the final distortion and residual stresses of gas turbine engine parts to optimize the manufacturing process.
\end{abstract}

\section{Introduction}

Additive Manufacturing enables manufacturing of parts by means of additive layer synthesis and thus getting complex topology parts. From the time AM technologies came into use significant progress has been achieved in understanding the processes, structure and properties of the parts being made. The objective of this work is to generate a set of mathematical models and user environment (APDL program set) based on ANSYS Finite Element Analysis System solver which would allow performing, with the required degree of accuracy, the technological operation of numeric prediction of manufacturing and residual stress fields formation and displacements in the process of Selective Laser Melting of gas turbine engine components blanks for further optimization of manufacturing process parameters.

\section{Development}

The chosen simulation concept is based on the elements "animation" technique involving natural (unstrained) condition of the built-up part of material at the time it emerges. The area occupied by the finished product and powder at the final building stage is considered as design area. Continuous build-up of the metal (active) and support structure is performed discretely, at each sub-stage of computation corresponding to the "birth" of each next

\footnotetext{
*Corresponding author : dubrovskaya-as@avid.ru
} 
subdomain from the "dead" elements, the boundary task of heat transfer is solved, and the result of the previous sub-stage solution serves as initial conditions for the subsequent one.

The verification of the developed numerical algorithms was carried out based on experimental data (Fig. 1). Comparison of analysis results for several discrete analogs of the process with different level of detail is performed. The first group comprises finite element analogs with maximum level of virtual prototype detail. It addresses a full set of the materials participating in the process and the relevant geometrically isolated subareas: metal, powder, baseplate. The finite-element discretization pattern is shown in Fig. 2. In the figure, the red zone indicates the platform area, purple is for powder, green is the area occupied by the metal of the product.

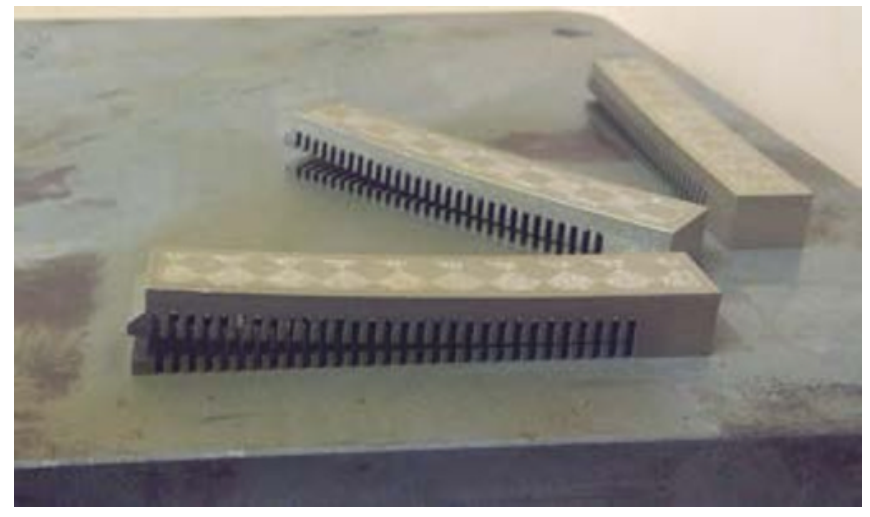

Fig. 1. Prototypes after Partial Cutting

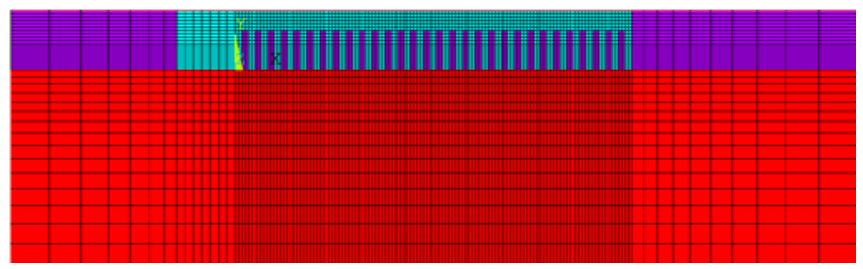

Fig. 2. Finite Element Model.

The tasks of unsteady heat transfer with moving boundaries and structural quasi-steady flow analysis are addressed successively. Mathematical substantiation of equations for recalculation of heat transfer parameters ensuring initial balance of heat energy is performed while switching to oversimplified spatiotemporal zoning unavoidable in numerical analysis. The comparison of 2D and 3D solutions with experimental data for the full-size test model demonstrated adequacy of assumed hypotheses. The next level of simulation involved averaging of thermomechanical and thermophysical properties of the supporting part of the structure.

The analysis of the obtained consistent patterns of temperature fields formation in the SLM process served the basis to develop an efficient analytical model of the next level which excludes powder and baseplate zones from consideration. Besides, heat transfer task solution is lacking, and the temperature in the structural task is set according to a special law ensuring minimum loss of accuracy. Fig. 3 shows the residual vertical deflection of the upper face of the samples obtained from the experiment, and its comparison with the design deflection. 


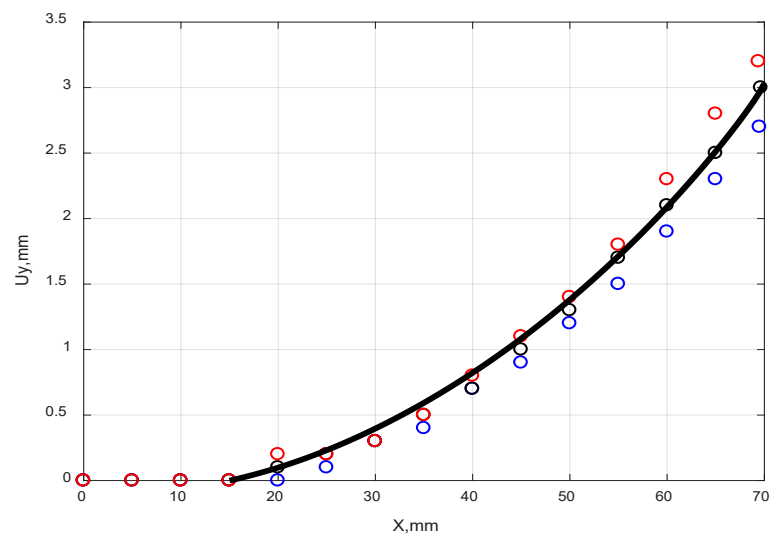

Fig. 3. Residual Vertical Deflection of the Samples Upper Face, mm. Line - estimated (black - full model, red - simplified); dots - experiment: blue marker - Sample 1, black - Sample 2, red - Sample 3.

\section{Mathematical statement}

The problem of determining the evolution of the system stress-strain state in the conditions of building up by selective laser melting (SLM) may be divided in 2 successive problems: unsteady heat conduction and quasi-steady problem of deformable solids mechanics which uses temperature profiles found at the first stage as volumetric loads. It is assumed that stress and strain arising in the system affect the heat balance.

The temperature and mechanical problems are addressed using the method of "killing" and further "revivification" (Elements Birth and Death in ANSYS) of part of material initially lacking in the model and then emerging in the process of applying the next powder layer and its local melting. The domain of computation is considered to be the area occupied by the already finished product and powder at the final stage of buildup. Continuous buildup of metal (working) and backup zones is performed discretely at each sub-phase of the computation corresponding to "revivification" of the next subdomain from "dead" elements, the boundary problem of heat conduction is solved, and the result of previous sub-phase solution serves as initial conditions for the next one.

At the $k^{\text {th }}$ sub-phase of solving, the statement of the boundary problem of unsteady heat conduction to determine $T(\mathbf{x}, t)$ temperature profiles in $V_{k}$ domain with $S_{k}$ boundary the heat conductance equation has the following form:

$$
\rho(\mathbf{x}) c(\mathbf{x}, T) \frac{\partial T}{\partial t}=\operatorname{div}(\lambda(\mathbf{x}, T) \operatorname{grad}(T))+\rho(\mathbf{x}) \dot{q}(\mathbf{x}, t) \mathbf{x} \in V_{k},
$$

where $c(\mathbf{x}, T), \lambda(\mathbf{x}, T), \rho(\mathbf{x})$ - heat capacity, heat conductance and density of the nonuniformly alloyed material respectively, $\dot{q}(\mathbf{x}, t)$ - external heat source specific capacity.

Boundary conditions:

$$
\lambda(\mathbf{x}, T) \operatorname{grad}(T) \cdot \mathbf{n}=h(T) \cdot\left(T-T_{c}(t)\right)+\varepsilon \sigma_{0}(T)^{4} \quad \mathbf{x} \in S_{k},
$$


where the first summand of the right part describes convective heat transfer, and the second one - radiation (Stefan-Boltzmann law); $\varepsilon$ - emissivity, $\sigma_{0}$ - Stefan-Boltzmann constant, $h(T)$ - heat transfer factor, $T_{c}(t)$ - ambient temperature, $\mathbf{n}$ - external unit normal to $S$ boundary of the cooled solid.

Initial conditions:

$$
T\left(\mathbf{x}, t_{0, k}\right)=T_{k-1}(\mathbf{x}), \quad \mathbf{x} \in V_{k},
$$

where $T\left(\mathbf{x}, t_{0, k}\right)$ - initial temperature distribution for the $k^{\text {th }}$ sub-phase, $T_{k-1}(\mathbf{x})$ temperature determined at the end of the previous one.

The uncoupled quasi-steady problem of deformable solids mechanics assuming insignificancy of mass forces contribution has the following form at the $k^{\text {th }}$ sub-phase:

Balance equation:

$$
\operatorname{div} \hat{\sigma}=0, \quad \mathbf{x} \in V_{k}
$$

where $\hat{\sigma}(\mathbf{x}, t)$ - stress tensor.

Cauchy geometrical relationships:

$$
\hat{\varepsilon}=\frac{1}{2}\left(\nabla \mathbf{u}+(\nabla \mathbf{u})^{T}\right), \quad \mathbf{x} \in V_{k},
$$

where $\mathbf{u}(\mathbf{x}, t)$ - displacement vector, $\hat{\varepsilon}(\mathbf{x}, t)$ - total strain tensor.

Boundary conditions in displacements:

$$
\mathbf{u}=\mathbf{U}, \quad \mathbf{x} \in S_{u, k},
$$

and stress

$$
\hat{\sigma} \cdot \mathbf{n}=\mathbf{P}, \quad \mathbf{x} \in S_{\sigma, k},
$$

where $S_{u}, S_{\sigma}$ - parts of the boundary with specified displacements and loads respectively.

Material thermomechanical parameters in the zone of "dead" elements exclude physical non-linearity, and are ideally elastic with degraded values:

$$
{ }^{4} C(\mathbf{x}), \mathbf{x} \in V_{k}^{k i l}<<{ }^{4} C(\mathbf{x}, T), \mathbf{x} \in V_{k}^{k i v}
$$

where ${ }^{4} C$ - rank 4 tensor of material elastic constants.

The general system of equations of the deformable solids mechanics boundary problem also includes defining relationships. Viscoelastoplastic behavior of the alloy of which the product is made taking into account the temperature range including the phase change envisages the possibility of using several different physical models of plasticity. The plasticity model with bilinear isotropic hardening and temperature dependence of parameters is taken as the baseline model. Material behavior in the equivalent stressequivalent strain coordinates is shown in Figure 4. 


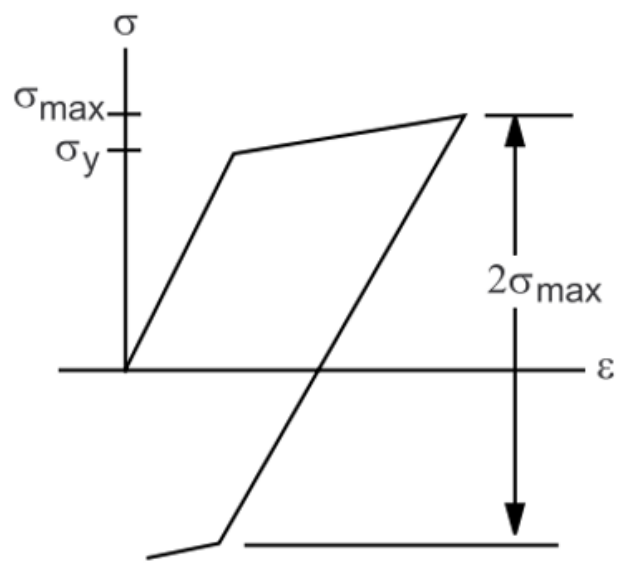

Fig. 4. Diagram $\sigma-\varepsilon$ for bilinear isotropic model BISO

In Figure 4 the $\sigma_{y}$ value corresponds to the yield strength

\section{Adaptation}

For complex topology parts the algorithm of laminated voxel mesh building was developed in APDL. The developed and implemented system of simplification hypotheses enabled significant reduction of computational effort without considerable loss of accuracy. For verification of the developed program suite, simulation of the physical process of the typical aircraft engine part additive manufacturing was performed (Fig. 5). The results of mathematical modeling (Fig. 6) are compared with the real production process data for a given part.

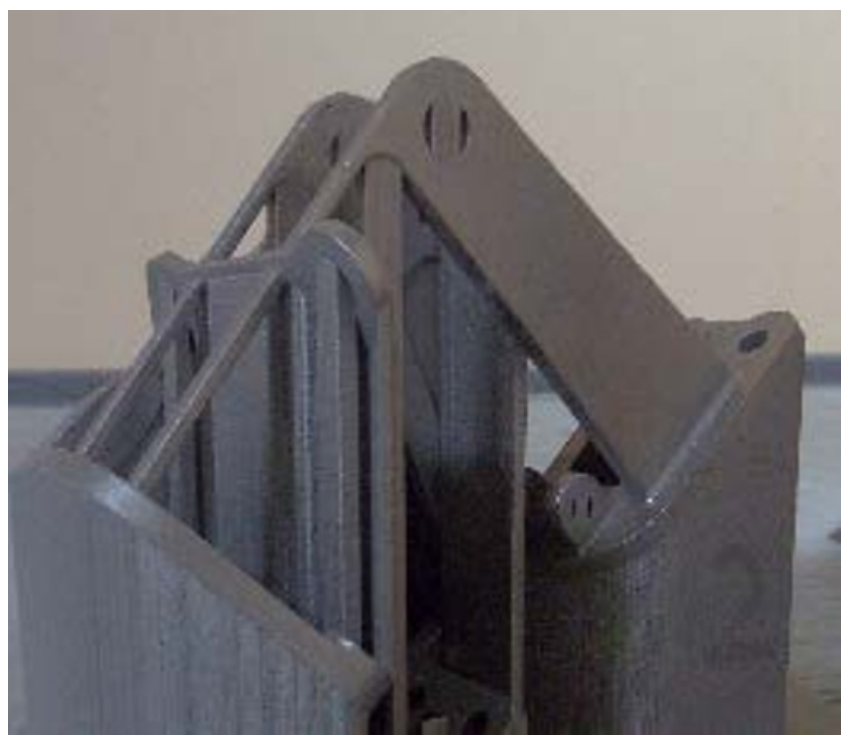

Fig. 5. Manufactured Part 


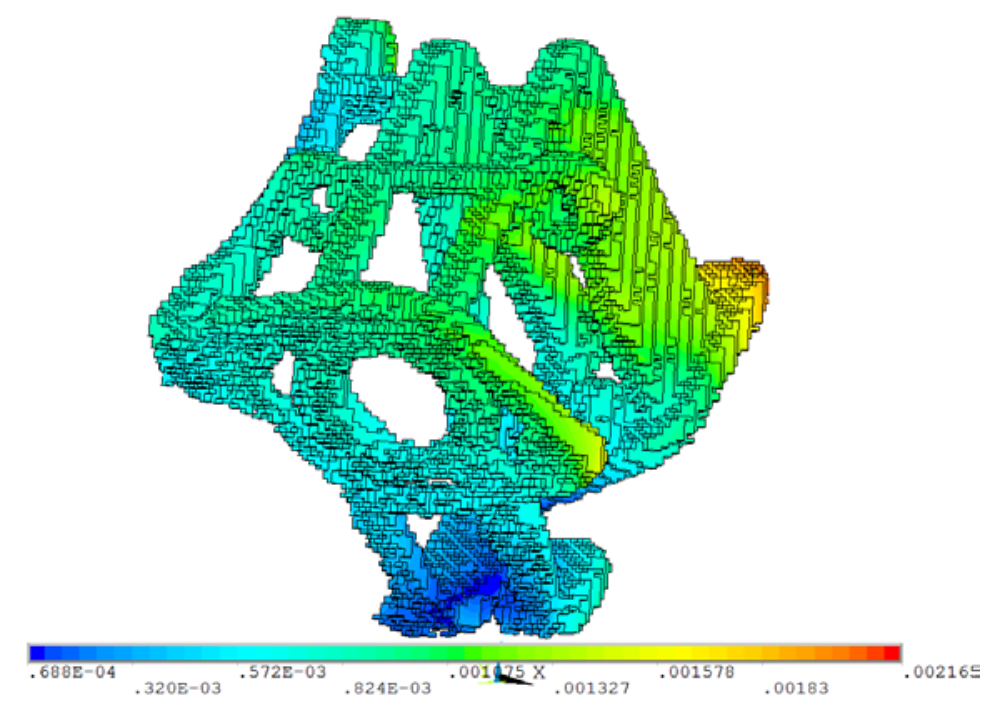

Fig. 6. Simulated Distortion

Simulation accuracy inspection was performed by comparing estimated distortion and measurement results from optical CMM for a 3D coordinate measurement. Fig. 7 shows the distortion of manufactured part based on 3D coordinate measurement results and Fig. 8 shows the same geometry comparison for simulation results.

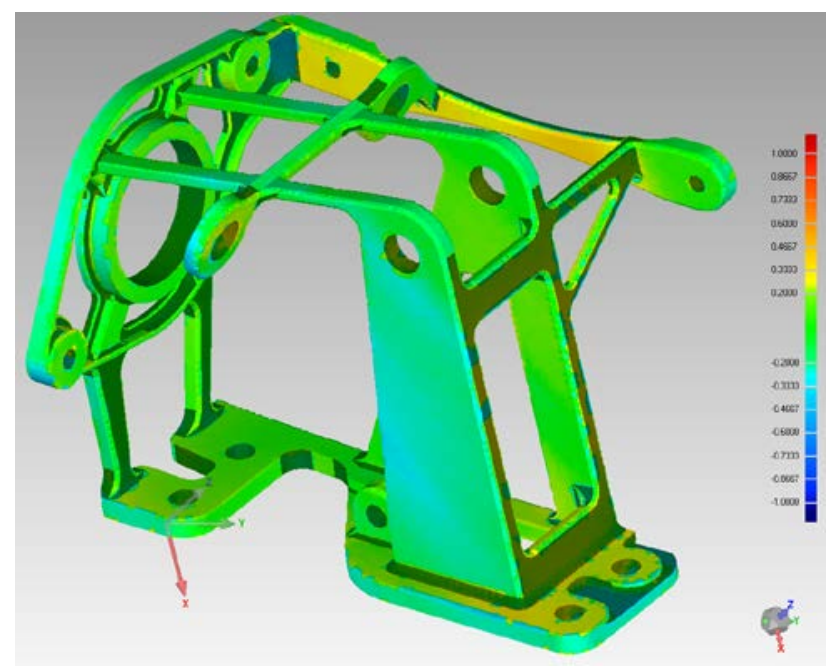

Fig. 7. Real manufactured part geometry control 


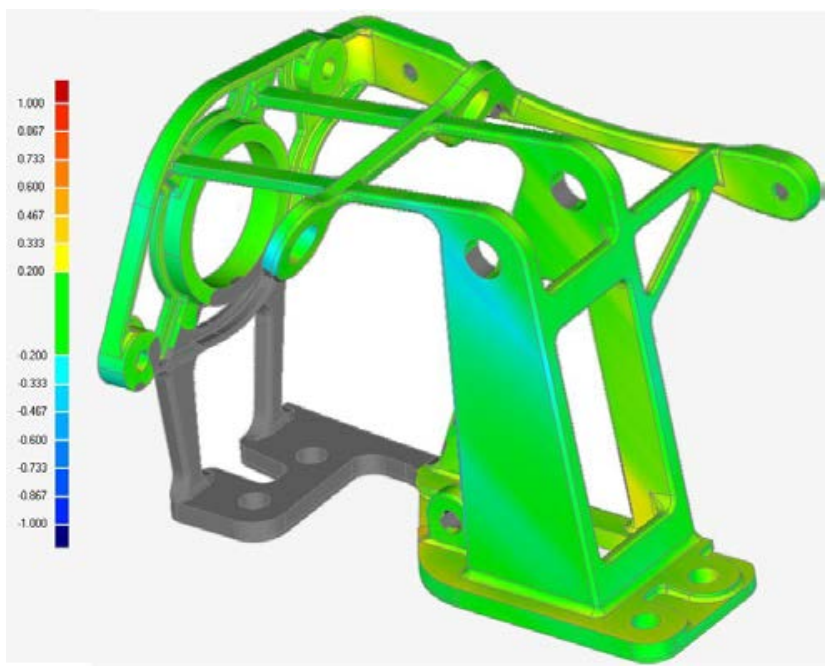

Fig. 8. Simulation results verification

The maximum absolute deviation of the simulation results against measurements was $0.19 \mathrm{~mm}$.

\section{Conclusion}

The objective of this work was to generate a set of mathematical models and user environment (APDL program set) based on ANSYS Finite Element Analysis System solver which would allow performing, with the required degree of accuracy, the technological operation of numeric prediction of manufacturing and residual stress fields formation and displacements in the process of Selective Laser Melting of gas turbine engine components blanks for further optimization of manufacturing process parameters. The developed and implemented system of simplification hypotheses enabled significant reduction of computational effort without considerable loss of accuracy. For verification of the developed program suite, simulation of the physical process of the typical aircraft part additive manufacturing was performed. The results of mathematical simulation were matched with this part actual manufacturing process data sourced from UEC-Aviadvigatel. Simulation accuracy was verified by comparing design strain and results of measurement by the optical measurement system for 3D coordinate measurement. The maximum absolute deviation of the simulation results against measurements was $0.19 \mathrm{~mm}$. Thus developed mathematical models and user environment are valid for prediction of distortion in Additive Manufacturing production preparation.

This work was performed with financial support from Ministry of Education and Science of the Russian Federation (contract No. 02.G25.31.0168 dated 01.12.2015) within implementation of the RF Government Order No. 218 «On the measures of the State support for development of higher education institutions and organizations implementing integrated projects on high-tech manufacturing build-up»». 


\section{References}

1. B. Brown, K.H. Kim, L. Anand An internal variable constitutive model for hot working of metals, International Journal of Plasticity, Vol. 5. pp. 95-130, 1989.

2. R.N. Faskhutdinov, A.S.Dubrovskaya, K.A. Dongauzer, P.V. Maksimov and N A Trufanov Topology optimization of a gas-turbine engine part, IOP conference series: materials science and engineering, №177, 012077, 5p, 2017.

3. A. Dubrovskaya, K. Dongauzer and R. Faskhutdinov The design of lightweight gas turbine engine parts using topology optimization, MATEC Web of Conferences, Vol. 129, 4p, 2017.

4. S.A. Khairallah, A.T. Anderson, A. Rubenckik, and W.E. King Laser powder-bed fusion additive manufacturing: Physics of complex melt flow and formation mechanisms of pores, spatter and denudation zones, Acta Materialia, pp. 108:36-45, 2016.

5. . Ding, P. Calegrove, J. Mehnen, P.M. Sequeira Almeida, F. Wang, and S. Williams. Thermo-mechanical analysis of Wire and Arc Additive Layer Manufacturing process on large multi-layer parts. Computational Materials Science, 50, pp. 3315-3322, 2011.

6. N. Hodge, R. M. Ferencz, J. M. Solberg Implementation of a thermomechanical model for the simulation of selective laser melting, Computational Mechanics, Vol. 54, Issue 1, pp 33-51, 2014.

7. W. King, A. Anderson, R. Ferencz, N. Hodge, C. Kamath, S. Khairallah Overview of modelling and simulation of metal powder bed fusion process at Lawrence Livermore National Laboratory, Material Science and Technology, 31(8), pp.957-968, 2015.

8. S. Kolossov, E, Boillat 3D FE simulation for temperature evolution in the selective laser sintering process, International Journal of Machine Tools and Manufacture, 44 (2-3), pp. 117-123, 2004.

9. R. B. Patil, V. Yadava. Finite element analysis of temperature distribution in single metallic powder layer during metal laser sintering, International Journal of Machine 